\title{
Implementation of face and eye detection on DM6437 board using simulink model
}

\author{
B. Vijayalaxmi ${ }^{1}$, Kaushik Sekaran ${ }^{2}$, N. Neelima ${ }^{3}$, P. Chandana ${ }^{4}$, Maytham N. Meqdad ${ }^{5}$, \\ Seifedine Kadry ${ }^{6}$ \\ ${ }^{1,2,4}$ Vignan Institute of Technology \& Science, Hyderabad, India \\ ${ }^{3}$ CMR Institute of Technology, Hyderabad, India \\ ${ }^{5}$ Al-Mustaqbal University College, Hillah, Babil, Iraq \\ ${ }^{6}$ Department of Mathematics and Computer Science, Faculty of Science, Beirut Arab University, Lebanon
}

\begin{tabular}{|c|c|}
\hline Article Info & ABSTRACT \\
\hline & \multirow{9}{*}{$\begin{array}{l}\text { Driver Assistance system is significant in drriver drowsiness to avoid on road } \\
\text { accidents. The aim of this research work is to detect the position of driver's } \\
\text { eye for fatigue estimation. It is not unusual to see vehicles moving around } \\
\text { even during the nights. In such circumstances there will be very high } \\
\text { probability that a driver gets drowsy which may lead to fatal accidents. } \\
\text { Providing a solution to this problem has become a motivating factor for this } \\
\text { research, which aims at detecting driver fatigue. This research concentrates } \\
\text { on locatingthe eye region failing which a warning signal is generated so as } \\
\text { to alert the driver. In this paper, an efficient algorithm is proposed } \\
\text { for detecting the location of an eye, which forms an invaluable insight } \\
\text { for driver fatigue detection after the face detection stage. After detecting } \\
\text { the eyes, eye tracking for input videos has to be achieved so that the blink } \\
\text { rate of eyes can be determined. }\end{array}$} \\
\hline Received Aug 14, 2019 & \\
\hline Revised 30 Dec, 2019 & \\
\hline Accepted Feb 15, 2020 & \\
\hline Keywords: & \\
\hline Driver assistance system & \\
\hline Eye region & \\
\hline Fatigue detection & \\
\hline Image & \\
\hline
\end{tabular}

Processing

Tracking

This is an open access article under the CC BY-SA license.

\section{Corresponding Author:}

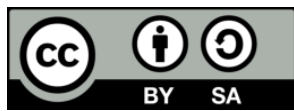

Seifedine Kadry,

Department of Mathematics and Computer Science,

Faculty of Science, Beirut Arab University, Lebanon.

Email: s.kadry@bau.edu.lb

\section{INTRODUCTION}

Driver drowsiness is a major factor in more number of road accidents. According to recent statistics annually nearly 1,200 deaths and 76,000 injuries are related to driver drowsiness. Driver assistant system is one solution to prevent these on road accidents, which is a major challenge. New technologies need to be developed to avoid these hazards on road [1]. The aim of this research work is to develop a prototype of driver assistance [2] system. The proposed algorithm focuses on designing a system, which accurately monitors the status of driver's eyes. Drowsiness of driver is first observed in eyes; hence the algorithm is designed to monitor the driver's eyes to avoid on road accidents. Drowsiness detection involves sequence of steps such that detection of face, observation of eye movements and eye blink rate. This research work focuses on tracking of eyes looking at the face of the image captured using an efficient image-processing algorithm. After successful detection of eyes form the capture image, the system is designed to know the status of the eyes to detect driver drowsiness. Most of the applications such as facial recognition, accident avoidance systems, virtual tools and human identification systems involve the analysis of face images.

Some specific applications may be listed as:

a. Driver monitoring systems: Analysis of visual attention is an important parameter to avoid road accidents. Hence, monitoring status of eyes and gaze detection can be used to avoid on road accidents. 
b. Marketing and Advertising: In print and advertising media eye tracking can provide insight into how much visual attention the consumer pays in different forms.

c. Interactive information systems: Eye movement based informational display systems in which the user uses his/her eyes as a pointing device such as a mouse pointer to navigate visually selectable items.

d. Security systems based on iris recognition.

e. Clinical diagnostics.

f. Computer gaming.

The aim of research work is to detect driver's drowsiness level. The focus will be placed on designing a system that will accurately monitor the status of driver's eyes. Many existing research work is referred while developing this proposed algorithm. Drowsiness Detection involves a sequence of steps such as detection of face from the captured images, identification of eyes and eye location and blink patterns. This research work focuses on the localization of the eyes, which involves looking at the entire image of the face, and determining the position of the eyes, by a self developed image-processing algorithm. The system is carried out in following steps.
a. Face detection
b. Eye detection
c. Eye tracking
d. Fatigue detection

\section{RESEARCH METHOD}

The implementation of status of eyes using MATLAB [3,4] is the major part of this research work. This was done to minimize size and improve efficiency of the code. The entire algorithm is focused on locating eyes, which involves looking at the entire image of the face and determining the status of the eyes. The eye blink rate-monitoring program can be divided up into a number of sections like face extraction, face detection, eye detection, and eye tracking. The performance of each step is highly dependent on the steps before itself because the results obtained in each component is passed as an input to the next component Figure 1.

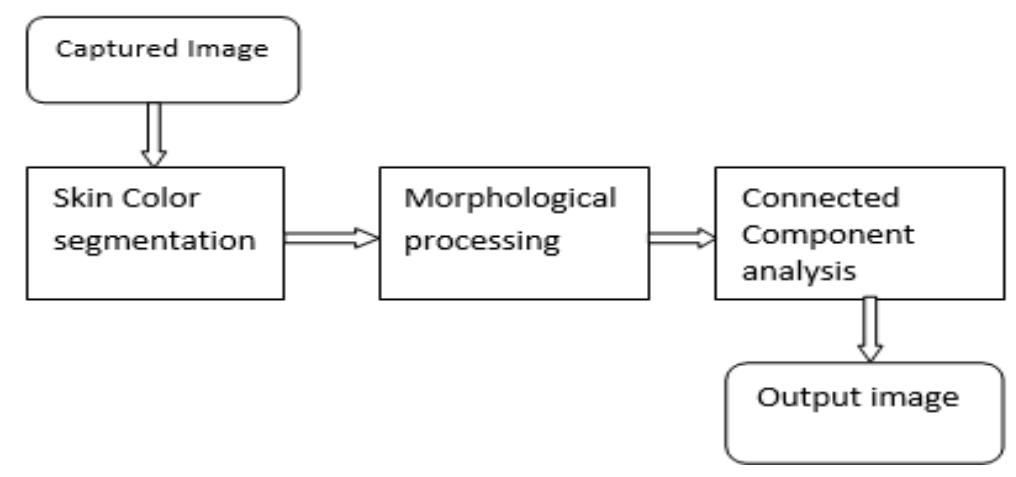

Figure 1. Architecture block diagram

\subsection{Face extraction}

Face Detection: For the captured image, the aim of face detection [5] is to detect the existence of face in the image. If the face is present in the image, return the image location with face detection. Detection of eye: For the captured image, the aim of eye detection is to detect the eyes. Eye detection algorithm is designed to locate the eyes and mark them with a square or rectangle mark.

\subsection{Face extraction mechanism}

Face extraction mechanism involves, identification and detection of face from the captured sequence of images. The common approach of face detection is to detect the face region by using the characteristic of the skin color.

\subsection{Algorithm for face extraction}

a. Convert the captured RGB image into $\mathrm{YCbCr}$ image. 
b. For each pixel, get the corresponding $\mathrm{Y}, \mathrm{Cb}$ and $\mathrm{Cr}$ values; in order to use them with skin thresholding, depending on the $\mathrm{Y}$ value for skin $\operatorname{col}$ or $(50<\mathrm{Y}<142 ; 107<\mathrm{Cb}<124 ; 135<\mathrm{Cr}<152)$ as our proposed solution.

c. Find the different regions in the image, in this step two functions are used the open and fill functions; to ensure that only skin areas were selected, then apply connected component analysis, which will separate connected heads by using open and close operations

d. Set some threshold value to separate face and non-face object based on skin region.

e. For each region, if height and width are within the range then is a face else it is not a face.

f. It means depending on the dimension of the object not too much or very small $(500<$ Area $<2500)$, as in our proposed solution.

Code for face extraction is written in the Mat lab software using the above algorithm. The Simulink model is designed using the code written in matlab. Example of face extraction is given in Figure 2.

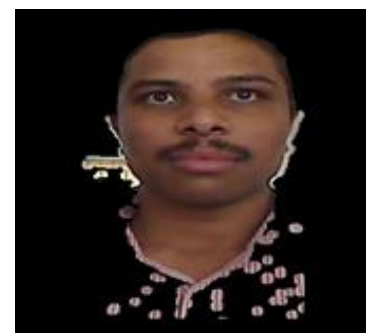

Figure 2. Extracted face

\subsection{Eye detection mechanism:}

a. Apply lab transform to the extracted face region.

b. Apply morphological operations on the output of lab transformed image.

c. Identify Region of interest (ROI)

d. Apply region properties on the eye pair

After successful extraction of face and eye regions from the captured image, eye tracking is performed. Therefore, eye location is an indispensable step for eye state detection in the driver fatigue $[6,7]$ monitoring system. At the first frame, eyes must be located before tracking, and to make sure that the correct feature is being tracked, and eyes should be relocated periodically during the tracking. Since we are taking only the face area, we know that the region of interest is the region containing the eyes. Divide the face into 6 quadrants; the region of eyes will be in uppermost 2 quadrants. After the ROI is extracted, lab transformation is applied on the eye pair and region properties like Bounding Box analysis are applied to detect the eyes through digital signal [8-10]. In the Bounding Box analysis the range of area of the eye component to be detected is $(100<\mathrm{Ar}<910)$ as in our proposed solution. Example of ROI and Eye detection is given in Figures 3 and 4 repectively. Code for ROI and eye detection is written in the MATLAB software using the above algorithm.
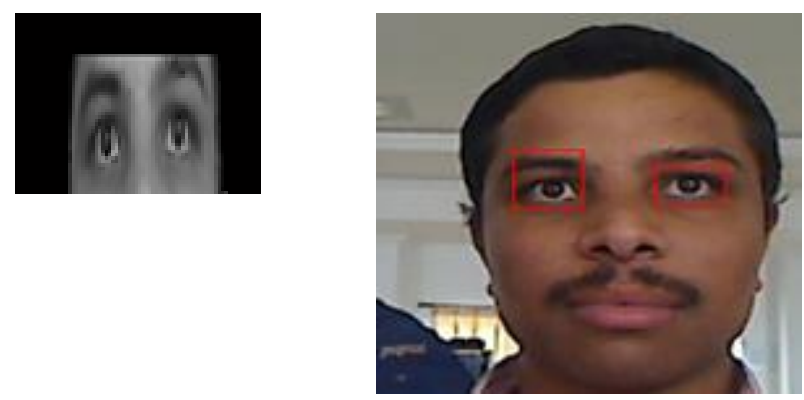

Figure 3. Result of ROI

Figure 4. Result of eye detection

\subsection{Fatigue detection}

The level of drowsiness is determined using the concept of fuzzy logic. The concept of fuzzy set is a class with un-sharp boundaries. It provides a basis for a qualitative approach to the analysis of complex 
systems in which linguistic rather than numerical variables are employed to describe system behavior and performance. It cannot be quantified objectively. Thus, we use computers to apply the fuzzy logic and determine the level of drowsiness. The mean and standard deviation are the two input variables used to calculate the status of the eyes. The physical state of the eye is the output variable.Results of state of the eye is given in Figures 5 and 6 respectively. Code to detect the state of the eye is written in the MATLAB software using the above algorithm. The standard deviation for the open eye is $(\mathrm{sd}<0.02)$ as in our proposed solution.

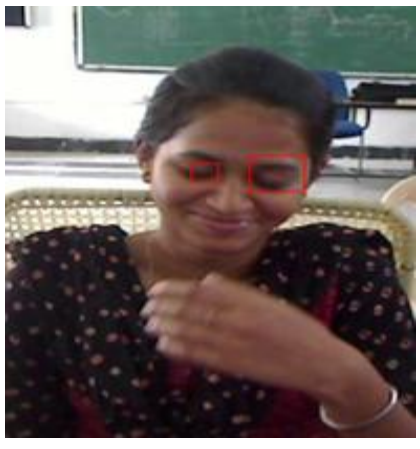

Figure 5. Closed eye

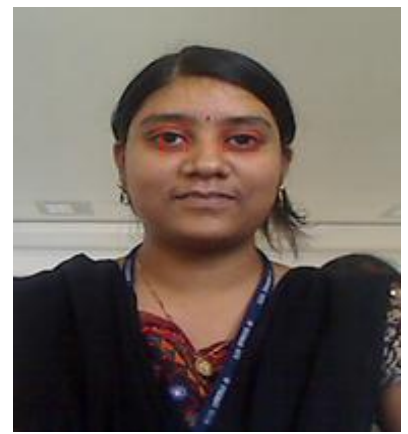

Figure 6. Open eye

\subsection{Code generation procedure for DM6437 EVM}

Code Generation procedure consists of MATLAB Simulink Software, CCSv4Software, and DM6437 EVM Library tool for the generation of code for the target. Code generation procedure steps for DM6437 EVM are shown in below flow chart figure 7.

Following are the steps to generate code for DM6437:

a. Open the Matlab/Simulink model, create the Simulink model for the required application

b. Run the model, create the make file and after creating the make file build the model and set the PSP driver path

c. If no errors, an .out file is generated.

d. Open the CCSv4 software, create the new configuration file and launch configuration file

e. Connect the target then load program i.e., .out file generated from MATLAB/SIMULINK model.

f. Run the code and check the output on the screen.

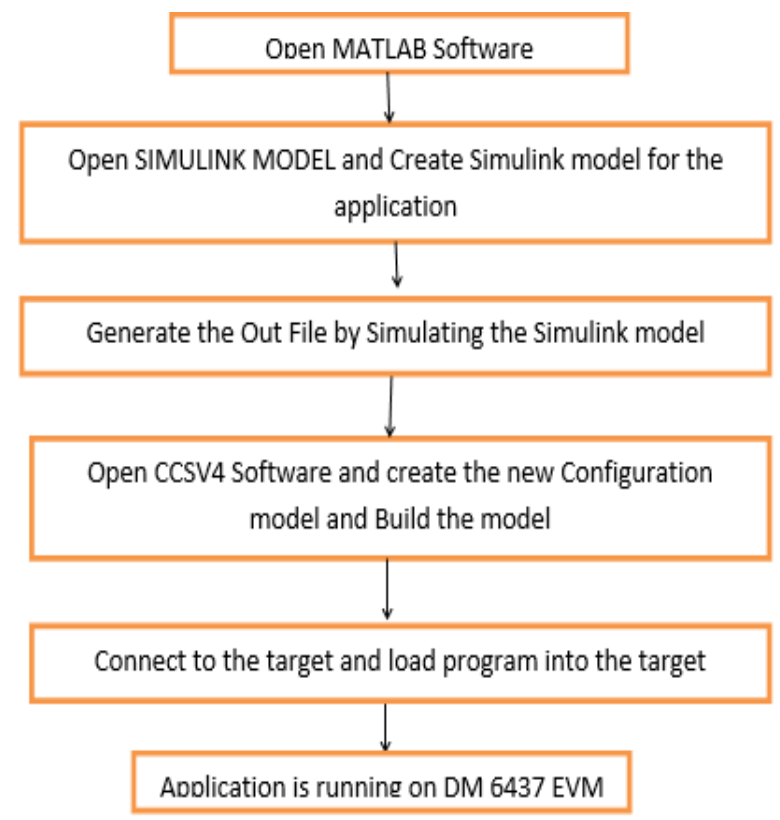

Figure 7. Code generation Procedure flow chart for DM 6437 EVM [11, 12] 


\section{RESULTS AND DISCUSSION}

This section discusses the results obtained from the SIMULINK model. This section also focuses on warning and testing procedures.

\subsection{Results analysis}

In this proposed algorithm, the continuous images are captured and relevant required information is extracted. The algorithm is designed to locate the eyes from the captured image and identify the status of the eyes. The entire algorithm is developed in MATLAB environment. SIMULINK model is developed with the required code generation to gain maximum benefit of code reused. The results of the software development are a method of tracking eyes when a video [13,14] is given as input to the Simulink model. The analysis if the algorithm results are given in Table 1.

Table 1. Result analysis

\begin{tabular}{ccccc}
\hline $\begin{array}{c}\text { S. } \\
\text { NO. }\end{array}$ & MEAN & $\begin{array}{c}\text { STANDERD } \\
\text { DEVIATION }\end{array}$ & $\begin{array}{c}\text { OUTPUT } \\
\text { RESULT }\end{array}$ & $\begin{array}{c}\text { STATUS OF } \\
\text { EYE }\end{array}$ \\
\hline 1. & 0.179 & 0.01742 & 0 & CLOSE \\
2. & 0.3366 & 0.2243 & 1 & OPEN \\
3. & 0.2931 & 0.0166 & 0 & CLOSE \\
4. & 0.2747 & 0.01384 & 0 & CLOSE \\
5. & 0.4611 & 0.05534 & 1 & OPEN \\
6. & 0.3534 & 0.01691 & 0 & CLOSE \\
7. & 0.3189 & 0.03361 & 1 & OPEN \\
8. & 0.1999 & 0.04755 & 1 & OPEN \\
9. & 0.3624 & 0.01552 & 0 & CLOSE \\
10. & 0.3281 & 0.03449 & 1 & OPEN \\
\hline
\end{tabular}

\subsubsection{The cases where our algorithm has failed}

In thid section, we will present three different cases where our algorithm failed case 1 shown in Figure 8. Reason behind the failure: The left eye part is not covered in the ROI as shown in the figure. The eyebrow that is covered is out of range of the area given. So only one eye is detected and the response time [15] could be calculated for the enhancement on the case of failure, case 2 Figure 9. Reason behind the failure: The right eye is covered in the ROI but its area is too small to be in the range. Hence, it is not detected, Case 3 Figure 10, Reason behind this failure: The right eyebrow that is covered in the ROI is in the range of the area given so the eyebrow also detected.
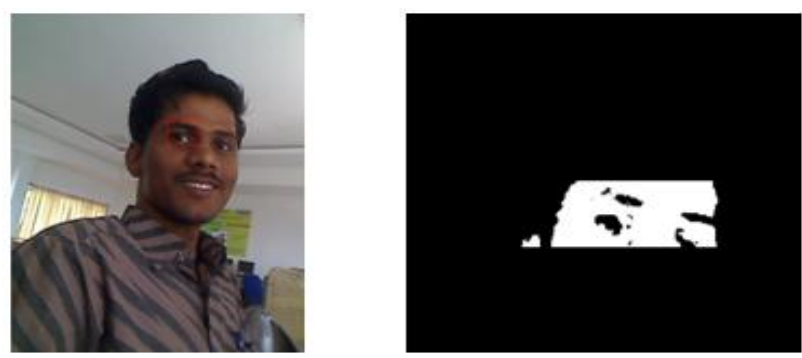

Figure 8. Failure case1
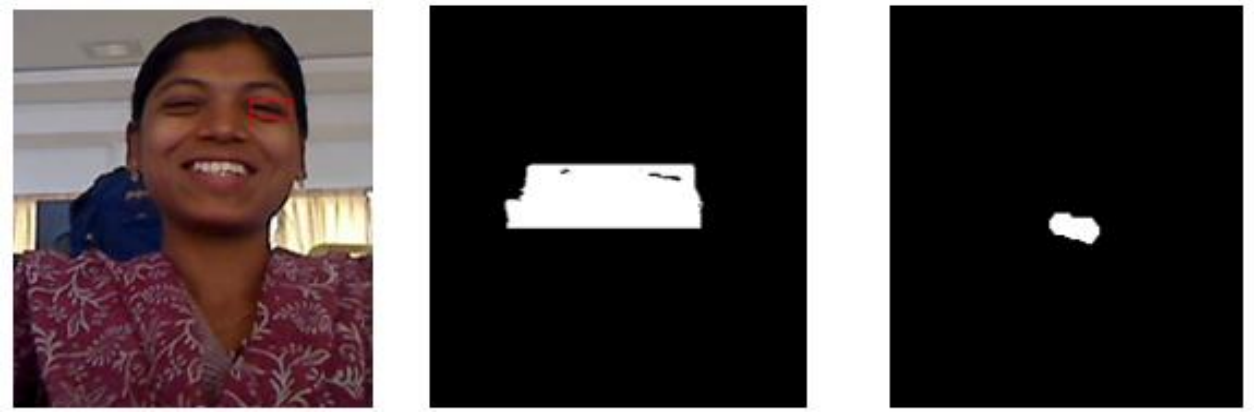

Figure 9. Failure case2 

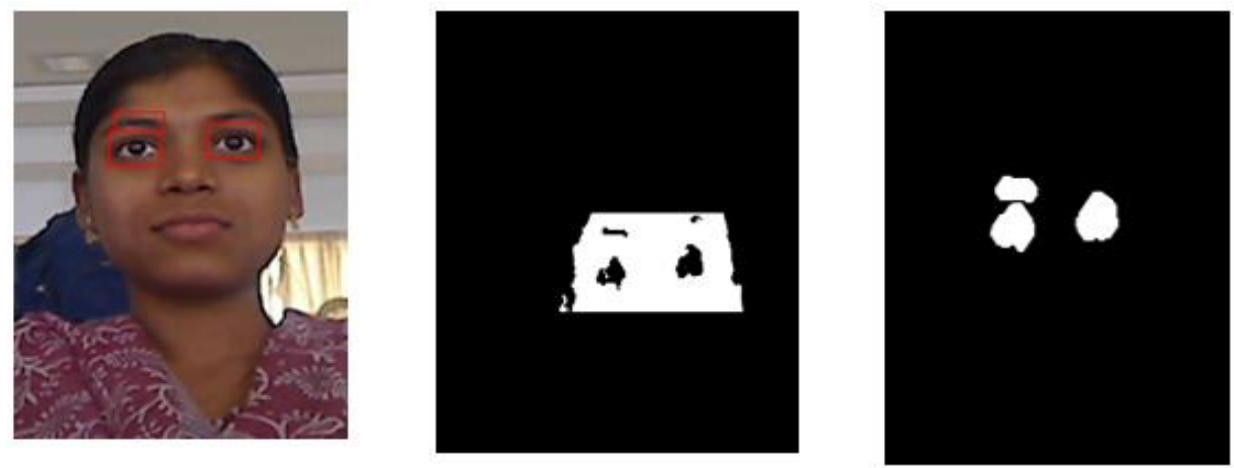

Figure 10. Failure case 3

\subsection{Alarm}

Alerting the drowsy driver: The main objective of the project is to alert the driver if the system decides that he has felt drowsy. To alert the drowsy driver $[16,17]$ we need an alarm. For this we've used an audio block at the output. It will give a beep sound if the state of the eye is closed. And if the state of the eye is open it will not give any sound. In this way the drowsiness level of the driver can be detected, and he can be alerted if he felt asleep [18-20].

\subsection{Testing}

Testing of each module is performed individually as the system is developed. The testing is done as a continuous process as the system is developed.

a. Matlab

Matlab code is tested after completion of each module. Each module is written as a part of function implementation. The errors can be easily identified through single stepping. The MATLAB code is tested for a continuous video, counting the number of correct detections versus the number of incorrect detections. This is done twice to check its effectiveness when implemented.

b. Overall system

The major difficulty faced is while testing the overall system, which resulted in delay in the system from different sections of code. Running the code using live video causes the processes in the region of 8 seconds to process a frame from capture to processed image output. This makes the system currently unworkable in real-time adding reaction delay to the scenario and the driver would have driven for approximately 10 seconds and could have easily gone off the road by this stage. The future work of this model is to store the eye extraction values in a public cloud [21-25] to fetch the data anywhere from the current place.

\section{CONCLUSION}

A new algorithm for Face and Eye state detection is implemented on DM6437 board using Simulink model. The proposed algorithm works on different images under different illumination conditions. Nearly 36 images from GTAV database 25 and 30 images from local database have been tested in the laboratory. The success rate of the proposed algorithm is $89.5 \%$. The purpose of this research work is to develop a driver fatigue detection system using vision-based approach based on eye blink rate. The algorithm is also tested on video directly connected to the board and results are quite promising, but certain cases mentioned in the analysis need to be improved. This paper presents a unique approach of designing Face and Eye detection using MATLAB/SIMULINK model which gives an opportunity to experience real-time hardware implementation. The analysis report on Video processing application shows that the total time required to process the Video is $0.33 \mathrm{~s}$, number of block methods for video are 9 , number of internal methods video are 5, number of model methods are for video are 10, the clock precision for video is 3 ns and clock speed is $3001 \mathrm{MHz}$. This paper presents an effective implementation of Face and Eye detection applications on DM6437 evaluation board for real time applications.

\section{ACKNOWLEDGEMENTS}

This paper is a part of research work on the project "Development of Non-Intrusive Driver Fatigue Detection \& Warning System to avoid on road Accidents" sanctioned under Early Career Research Award 
sponsored by Science \& Engineering Research Board, Govt. of India, New Delhi at Vignan Institute of Technology \& Science, Deshmukhi, Hyderabad.

\section{REFERENCES}

[1] Jackson, P., Hilditch, C., Holmes, A., Reed, N., Merat, N. \& Smith, L, "Fatigue and Road Safety: A Critical Analysis of Recent Evidence,'Road Safety Web Department for Transport, Publication, no. 21, 2011.

[2] P. Sudhakar Rao, Vijayalaxmi, S. Sreehari, "New procedure for Segmenting Eyes from human Face,"International Journal of Emerging Technologies and Applications in Engineering, Technology and Sciences, ISSN:0974-3588, vol 4, no. 2, 2011.

[3] Matlab/Simulink environment description, Mathworks Inc, [Online]. Available: https://www.mathworks.com/matlabcentral/answers/114571-how-to-detect-eyes-in-matlab, 2013.

[4] W. S. Gan and S. M. Kuo, "Transition from Simulink to MATLAB in Real-Time Digital Signal Processing Education," TheInternational Journal of Engineering Education, vol. 21, no. 4, pp. 587-595, 2005.

[5] Uno, H., "Detection Decline in Arousal Level Using Combined Physiological and Behavioral Measures," Japan Automobile Research Institute, vol. 25, no. 8, 2003.

[6] Karamjeet Singh, Rupinder Kaur, "Physical and Physiological Drowsiness Detection Methods,"International Journal of IT, Engineering and Applied Sciences Research, vol. 2, vol. 34-43, 2013.

[7] Elsenbruch,S., Harnish,M., and Orr, W.C, "Heart rate variability during waking and sleep in healthy males and females," Sleep, vol. 22, pp. 1067-1071, 1999.

[8] V. K. Ingle and J. G. Proakis, "A DSP Course based on Lecture/ Lab Integration," IEEE Signal Processing Magazine, vol. 9, no. 4, pp. 25-29, Oct. 1992.

[9] R. Chassaing, W. Anakwa, and A. Richardson, "Real-Time Digital Signal Processing in Education," in Proc. Int. Conf.on Acoustics, Speech and Signal Processing (ICASSP), Minneapolis, USA, IEEE, vol. 1, pp. 28-31, Apr 1993.

[10] Li Shuo, Long Teng, Li Fanghui and Tian Liyu, "Real-time signal waveform library and processing system based on TMS320C5x DSP Starter Kit," ICSP '98. 1998 Fourth International Conference on Signal Processing (Cat. No.98TH8344), Beijing, China, vol. 2, pp. 1662-1665, 1998.

[11] Salami Ifedapo Abdullahi, Mohamed Hadi Habaebi, Noreha Abd Malik, "Design, simulation and practical experimentation of miniaturized turbine flow sensor for flow meter assessment," Bulletin of Electrical Engineering and Informatics, vol. 8, no.3, pp. 777-788, Sep 2019.

[12] TMS320DM6437, "Evaluation Module with TVP5146M2,"Texas Instruments, [Online]. Available: http://www.ti.com/lit/ug/spruev6/spruev6.pdf, 2007.

[13] T. Marciniak, D. Jackowski, P. Pawłowski, and A. Da browski, "Realtime people tracking using DM6437 EVM," in Proc. IEEE Signal Processing Algorithms, Architectures, Arrangements, and Applications Conference Proceedings, Poland Sect, (SPA'2009), Pozna'n, Poland, pp. 116-120,Sep 2009.

[14] J. Balcerek, A. Chmielewska, A. Da browski, D. Jackowski, A. Konieczka, T. Marciniak, and P. Pawłowski, "Recognition of threats in urban areas by means of the analysis of video sequences," in Proc. IEEE Multimedia Communications, Services and Security (MCSS’2010), Kraków, Poland, pp. 41-48, May 2010.

[15] McCall,J., and Trivedi,M., "Video-Based Lane Estimation and Tracking for Driver Assistance: Survey, System, and Evaluation," IEEE Trans. Intelligent Transportation Systems, vol. 7, no. 1, 2006.

[16] Sekaran, Kaushik, Mohammed S. Khan, Rizwan Patan, Amir H. Gandomi, Venkata Krishna Parimala, and Suresh Kallam. "Improving the Response Time of M-Learning and Cloud Computing Environments Using a Dominant Firefly Approach,” DOI: 10.1109/ACCESS.2019.2896253, IEEE Access, vol. 7, pp. 30203-30212, 2019.

[17] Eoh,H.J., Chung,M.K, and Kim,S.H., "Electroencephalographic study of drowsiness in simulated driven with sleep deprivation," International Journal of Industrial Ergonomics, vol 35, pp.307-320, 2005.

[18] Lal,S.K.L., Craig,A., Boord,P., Kirkup,L., and Nguyen,H., "Development of an algorithm for an EEG-based driver fatigue countermeasure," Journal of Safety Research, vol 34, pp. 321-328, 2003.

[19] Toscani,L., Gangemi,P.F., Parigi,A., Silipo,R., Ragghianti, P., Sirabella,E., Morelli, M.,Bagnoli,L., Vergassola,R., and Zaccara,G., "Human heart rate variability and sleep stages," The Italian Journal of Neurological Sciences, vol 17, pp. 437-439, 1996.

[20] Bonnet, M.H., and Arand, D.L.,"Heart rate variability: sleep stage, time of night, and arousal influences," Electroencephalography and Clinical Neurophysiology, vol 102, pp.390-396, 1997.

[21] Tsunoda, M., Endo,T., Hashimoto,S., Honma,S., and Honma, K.,I., "Effects of light and sleep stages on heart rate variability in humans," Psychiatry and Clinical Neurosciences, vol 55, pp. 285-286, 2001.

[22] Sekaran, Kaushik, and P. Venkata Krishna, "Big Cloud: a hybrid cloud model for secure data storage through cloud space,"International Journal of Advanced Intelligence Paradigms 8, no. 2, pp. 229-241, 2016.

[23] S Yassine, S Kadry, MA Sicilia, "A framework for learning analytics in moodle for assessing course outcomes," IEEE Global Engineering Education Conference (EDUCON), pp. 261-266, 2016.

[24] S Kadry, "Diagnostics and Prognostics of Engineering Systems: Methods and Techniques," IGI Global, vol. 1, paper. 461, 2012.

[25] S Kadry, R Younès, "Etude Probabiliste d'un Systeme Mecanique a Parametres Incertains par une Technique Basee sur la Methode de transformation,"Proceeding of CanCam, Canada 2005. 